\title{
Article \\ Measurement Method and Experimental Analysis of Liquid Entrainment for a Flooded Evaporator of a Water-Cooled Centrifugal Chiller Based on Energy Balance
}

\author{
Xinghua Huang ${ }^{1, * \mathbb{D}}$, Yunqian Zhang ${ }^{2,3}$, Zuqiang $\mathrm{Li}^{1}$ and Yaolin Lin ${ }^{4, *}$ \\ 1 Department of Thermal Energy and Power Engineering, Shanghai University of Engineering Science, \\ Shanghai 201620, China; m010119504@sues.edu.cn \\ 2 Chongqing Midea General Refrigeration Equipment Co., Ltd., Chongqing 401336, China; \\ zhangyq10@midea.com.cn \\ 3 School of Civil Engineering, Chongqing University, Chongqing 400044, China \\ 4 School of Environment and Architecture, University of Shanghai for Science and Technology, \\ Shanghai 200093, China \\ * Correspondence: xhhuang@sues.edu.cn (X.H.); yaolinlin@gmail.com (Y.L.); \\ Tel.: +86-13661531775 (X.H.); +86-13545064359 (Y.L.)
}

Citation: Huang, X.; Zhang, Y.; Li, Z.; Lin, Y. Measurement Method and Experimental Analysis of Liquid Entrainment for a Flooded Evaporator of a Water-Cooled Centrifugal Chiller Based on Energy Balance. Appl. Sci. 2021, 11, 8165. https://doi.org/10.3390/app11178165

Academic Editor: Francesca Scargiali

Received: 6 August 2021

Accepted: 30 August 2021

Published: 2 September 2021

Publisher's Note: MDPI stays neutral with regard to jurisdictional claims in published maps and institutional affiliations.

Copyright: (c) 2021 by the authors. Licensee MDPI, Basel, Switzerland. This article is an open access article distributed under the terms and conditions of the Creative Commons Attribution (CC BY) license (https:// creativecommons.org/licenses/by/ $4.0 /)$.

\begin{abstract}
Liquid entrainment in a flooded evaporator has an important impact on the performance and safety of a water-cooled centrifugal chiller. In this paper, two methods for measuring the liquid entrainment factor in the evaporator of a centrifugal chiller based on energy balance are proposed. Method 1 involves only the heat exchange capacity of the evaporator and Method 2 involves both evaporator and condenser. The applicable conditions of the methods are discussed. Experimental measurements on the flooded evaporator of a single-stage water-cooled centrifugal chiller with refrigerant R134a show that, for a system with good thermal balance, there is little difference in the entrainment factor values obtained by the two methods. Method 2 was found to have slightly higher measurement accuracy, compared to Method 1 . The uncertainty propagation analysis shows that for Method 2, the inlet and outlet water temperatures of the evaporator and condenser, motor input power, motor efficiency, transmission power loss and compressor suction and discharge temperatures are important factors. The experimental results show that the variation of the evaporator entrainment factor with refrigerant charge amount is different for different cooling capacity. At 700 and 800 refrigeration ton (RT), the entrainment factor of the test evaporator increases with the increase of refrigerant charge and the growth rate gradually accelerates. For the chiller tested, when the entrainment factor reaches $0.89 \%$ and $1.02 \%$, respectively, at 700 ton and 800 ton, the rapid increase of the entrainment factor leads to a significant decrease in the coefficient of performance (COP) during the charging process. Based on the analysis of the experimental results, it is recommended that the maximum entrainment factor for efficient operation of the centrifugal chiller should be controlled within $1 \%$.
\end{abstract}

Keywords: liquid entrainment; flooded evaporator; water-cooled centrifugal chiller; energy balance; experimental analysis

\section{Introduction}

Liquid entrainment/carryover is a common phenomenon for the shell-and-tube flooded or falling film evaporators of a water-cooled centrifugal chiller and has an important impact on the performance and safety of the chiller and compressor. Liquid entrainment reduces the amount of liquid that could otherwise be evaporated for refrigeration and reduces the cooling capacity. At the same time, it vaporizes in the compressor and consumes compressor power; therefore, it is detrimental to the chiller performance. In severe cases, the entrained liquid can erode and damage the impeller. In the boiling tube bundle of the flooded evaporator, liquid droplets are generated by the burst of bubbles 
near the liquid surface and the shearing effect of the vapor flow inside the tube bundle on the liquid ligament. When the liquid level is higher than the bundle, a liquid layer may exist above the bundle and the droplets may also be produced by the wave action of this layer. In addition, if the pressure difference through the outlet of the evaporator exceeds the suppression effect of gravity and surface tension, droplets may also be generated at the vapor-liquid interface under the evaporator outlet. After the liquid droplets are generated, they enter the headspace above the bundle, the larger liquid droplets fall back to the liquid surface under the gravity effect and the smaller liquid droplets are entrained by the vapor flow into the compressor to form liquid entrainment, as shown in Figure 1. The figure depicts the scenario where the liquid level is just above the tube bundle. For clarity, the bubbles under the liquid level are not shown. To leave enough space for vapor-liquid separation, the height of the tube bundle in an actual flooded evaporator is generally designed to be $50-60 \%$ of the shell diameter.

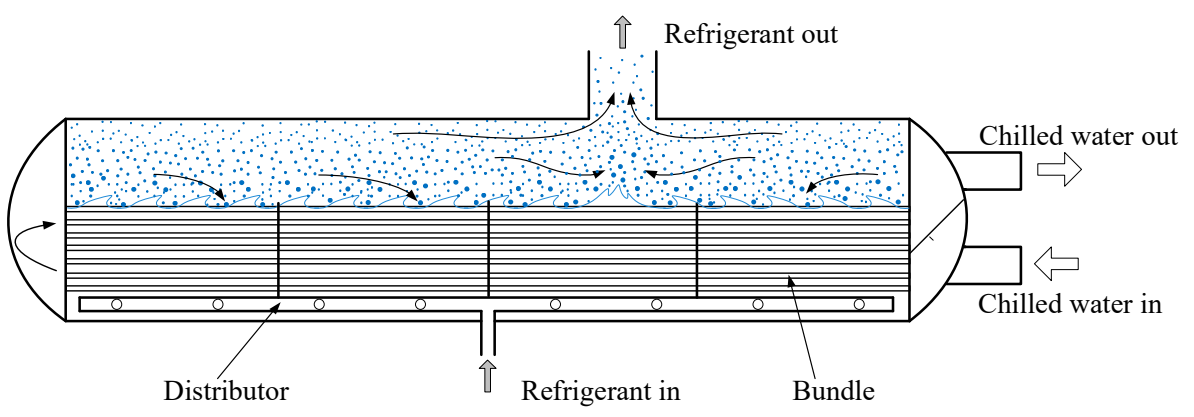

Figure 1. Schematic diagram of liquid entrainment in a flooded evaporator.

According to the different flow structure and liquid carryover mechanism, there are three types of liquid entrainment. The first type is liquid carryover by gas flow in straight tubes [1-4] and the second type is carryover through T-branches [5-7], the third category is the liquid entrainment in a liquid pool [8-10]. Liquid entrainment in straight tubes is caused by the shearing effect of the turbulent gas flow on the annular liquid film, while entrainment in the T-branches is caused by the destabilization of the gasliquid interface under the pressure difference between the main and branch tubes and entrainment in the pool is due to the boiling and bubbling gas flow from the liquid pool. The liquid entrainment problem in straight tubes [1-4] and T-branches [5-7] has received great attention with a large amount of experimental data having been accumulated and well-developed physical or semi-empirical models having been developed. However, there are relatively few studies on pool entrainment. Kataoka and Ishii [8] proposed a systematic pool entrainment model and classified pool entrainment into three regions, which are near-surface, momentum controlled and deposition-controlled regions. In each region, a specific correlation model is proposed to calculate the droplet entrainment factor. Due to the differences in the flow patterns in the pool droplet generation mechanisms, the momentum-controlled region is further divided into three sub-regions based on different levels of gas fluxes, but the exact form of entrainment correlation is not given when the gas flux is high. Sun et al. [9] experimentally studied the pool entrainment with side exit in the near-surface region. Zhang et al. [10] studied pool entrainment with and without side exit in the momentum-controlled region and proposed a new correlation with side exit in the high gas flux region. Lu and Xie [11] studied pool entrainment under small air flow rate and proposed an entrainment correlation at low air flow rate, with analysis on the effect of outlet location on the entrainment factor. All the above studies of pool entrainment are for air-water or steam-water systems.

The mechanism of liquid entrainment in the shell side of a flooded evaporator is complicated, including the entrainment mechanism both in the pool [8] and in the annular flow in horizontal tubes (when the liquid level is higher than the bundle) [1]. Therefore, the factors that affect the liquid entrainment in the pool and in the annular flow in hor- 
izontal tubes all have an impact on the liquid entrainment in the evaporator, including the cooling capacity and operating conditions of the unit, the height of the tube bundle and the refrigerant liquid level, the tube arrangement and the gaps between the tubes, the number of tube pass and the baffle structure in the headspace (if any), etc. For an actual shell-and-tube flooded or falling film evaporator, the mechanism is more complicated due to the three-dimensional characteristics of the vapor and droplets flow above the tube bundle (see Figure 1). However, there is only limited research on liquid entrainment in the evaporator with refrigerant. Using a laser and camera system and shadow graphic technique, Asher and Eckels investigated the distribution characteristics of droplets generated by the evaporation of refrigerant in the headspace of a rectangular tube bundle with R123 [12], R134a [13], respectively. The effects of heat flux, mass flow rate, evaporation temperature and refrigerant level on the liquid distribution generated in the headspace were investigated and the refrigerant level, mass flow rate and evaporation temperature were found to have the greatest influence. For the R134a refrigerant, computational fluid dynamic (CFD) simulation using Lagrange discrete phase model was also applied to evaluate and validate the liquid distribution in the headspace using experimentally fitted droplet velocity and diameter parameters as the setting conditions for the discrete phase [13]. For the actual evaporator of a water-cooled centrifugal chiller, no research work on the liquid entrainment has been reported to the best of the authors' knowledge.

Regarding the influence of liquid entrainment on the performance of centrifugal compressors, the current research conclusions are not consistent. Surendran and Kim [14] studied the effect of injecting water droplets in the inlet air for a single-stage centrifugal compressor by CFD, the results show that droplet evaporative cooling can reduce the specific work for a given pressure ratio and improve the aerodynamic efficiency of the compressor. Halbe et al. [15] studied the effect of liquid entrainment on the performance of a two-stage centrifugal compressor using R134a as the refrigerant. The heat, mass and momentum transfer between entrained droplets and vapor were simulated by the Eulerian-Lagrangian approach. The results show that the vapor flow field inside the compressor was altered by liquid carryover, making the compressor operated at off-design conditions. Meanwhile, the evaporation of droplets requires power consumption, which reduces the adiabatic and polytropic efficiency of the compressor, but the initial size of droplets entering the compressor has little effect on the efficiency. The effect of liquid entrainment on the efficiency of the centrifugal compressor may be related to the physical properties of working fluids, more experimental data are needed.

From the literature above, it can be concluded that there is little research on liquid entrainment in the evaporator of centrifugal chillers. To fill this gap, two methods to measure the liquid entrainment in the evaporator based on the energy balance of the chiller and compressor were proposed and the liquid entrainment factor of the flooded evaporator of a single-stage water-cooled centrifugal chiller was measured and analyzed. The experimental data includes the variation in entrainment factor and the COP of the chiller with the refrigerant charge at different cooling capacities under AHRI full load conditions [16].

\section{Materials and Methods}

\subsection{Measurement Method}

This method is suitable for single-stage centrifugal chillers, but can also be applied to two-stage chillers by simply bypassing the economizer of the two-stage unit and closing the secondary stage suction. Figure 2 shows the refrigerant flow chart of a single-stage centrifugal chiller. The sub-cooled liquid refrigerant flows out from the bottom of the condenser. Its main part enters the evaporator after being throttled by an orifice, where it is distributed axially by the distributor at the bottom of the evaporator. The vapor-liquid two-phase refrigerant flows upward across the tube bundle, absorbing the heat of the cold water in the tubes and evaporating. The temperature of the cold water decreases. The evaporated gaseous refrigerant and a small amount of entrained droplets flow out from 
the top outlet of the evaporator and are drawn into the compressor and compressed into high-pressure superheated vapor, then return to the condenser, where it is condensed by the cooling water in tubes and subcooled. In addition, there are two refrigerant auxiliary circuits, one of which enters the motor chamber after passing through a throttling orifice, which cools the motor and then flows back to the evaporator from the bottom of the motor chamber. There is also an orifice on the motor return pipe to make the pressure in the motor chamber slightly higher than that in the evaporator. In another auxiliary circuit, the refrigerant enters the oil cooler through a thermal expansion valve (TXV). It cools the lubricating oil of the transmission system and returns to the evaporator. The superheated vapor from the oil cooler enters the evaporator and mixes with the refrigerant vapor in the evaporator and then is drawn into the compressor.

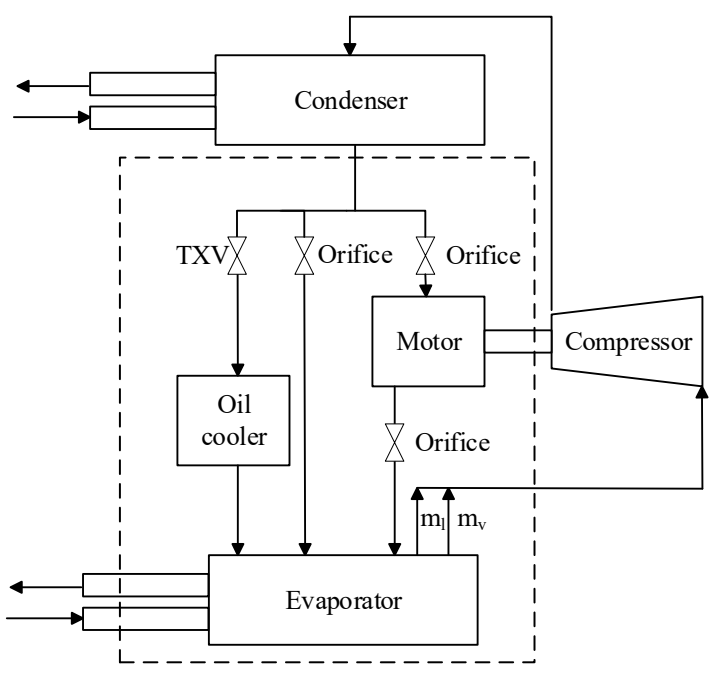

Figure 2. Refrigerant flow chart of a single-stage centrifugal chiller.

As shown in the dashed box in Figure 2, assuming that there is no hot gas bypass at the condenser outlet and ignoring the kinetic energy of the outlet liquid, the energy balance equation of the refrigerant is given by Equation (1).

$$
m_{v} h_{0, \text { evo }}+m_{l} h_{0, e l o}-\left(m_{v}+m_{l}\right) h_{\text {sub }}=Q_{e}+Q_{\text {motor }}+Q_{\text {oil }}
$$

where $m_{v}, m_{l}$ are the vapor and liquid flow rate from the evaporator, respectively; $m_{l}$ is the amount of liquid entrainment; $Q_{e}, Q_{\text {motor }}$ and $Q_{o i l}$ are the cooling capacity of evaporator, the heat dissipation rate of motor and the heat dissipation rate of oil cooler, respectively; $h_{0, \text { evo }}$ and $h_{0, e l o}$ are the total enthalpy of the refrigerant vapor and liquid at the outlet of the evaporator (i.e., the inlet of the compressor), respectively; $h_{\text {sub }}$ is the enthalpy of liquid refrigerant at the outlet of the condenser.

The total enthalpy of vapor $h_{0, e v o}$ and liquid droplets $h_{0, e l o}$ at the outlet of the evaporator can be calculated by Equations (2) and (3), respectively [17].

$$
\begin{aligned}
& h_{0, \text { evo }}=h_{\text {evo }}+\frac{u_{\text {evo }}^{2}}{2 \times 1000} \\
& h_{0, \text { elo }}=h_{\text {elo }}+\frac{u_{\text {elo }}^{2}}{2 \times 1000}
\end{aligned}
$$

where $h_{\text {evo }}$ is the enthalpy of refrigerant vapor at the evaporator outlet, determined by the suction pressure and temperature; $h_{\text {elo }}$ is the enthalpy of refrigerant saturated liquid at the evaporator outlet, determined by the suction temperature. $u_{e v o}, u_{e l o}$ is the vapor and droplets velocity, respectively. In Equations (2) and (3), 1000 is a unit conversion constant. It is assumed here that the velocity of droplets $u_{e l o}$ is equal to that of the vapor $u_{e v o}$, i.e., there is no slip between the vapor and liquid phase. Calculations show that when the 
droplet velocity varies from 0 to $u_{e v o}$, its impact on the liquid entrainment factor is less than $0.1 \%$.

Usually, a subcooling tube bundle is arranged at the bottom of the condenser and the outlet liquid enthalpy can be determined by pressure and temperature. It should be noted that for chillers with throttling orifices, a certain amount of refrigerant vapor may be entrained in the outlet of the condenser when the load is low. Due to the condensation heating of entrained hot vapor, the outlet subcooling is reduced or even no outlet subcooling. For the latter case, Equation (1) above is not applicable. The presence of refrigerant vapor can generally be determined by checking the subcooling degree at the condenser outlet or whether there are bubbles in the outlet pipe. For chillers with adjustable throttling flow area devices, such as floating ball valves, where the floating ball is above the lowest position, it can be considered that the condenser outlet is liquid refrigerant.

The evaporator cooling capacity $Q_{e}$ is calculated by Equation (4) according to the water temperature difference between the inlet and outlet and the water pressure drop.

$$
Q_{e}=\rho_{e w} V_{e w} c_{e w}\left(t_{e w i}-t_{e w o}\right)+V_{e w} \Delta p_{e w}
$$

Based on the energy balance of the motor, ignoring the heat dissipation from the motor's case, the heat dissipation of motor $Q_{\text {motor }}$ is equal to its power loss, as described by Equation (5).

$$
Q_{\text {motor }}=W_{\text {in }}\left(1-\eta_{e}\right)
$$

The transmission parts are cooled by the lubricating oil, which then dissipates heat to the refrigerant in the oil cooler, so the heat dissipation of the oil cooler $Q_{o i l}$ is equal to the power loss of the transmission parts $Q_{\text {mech }}$, given in Equation (6).

$$
Q_{\text {oil }}=Q_{\text {mech }}
$$

The energy balance of the compressor is described by Equation (7).

$$
\left(m_{v}+m_{l}\right) h_{0, c v o}-\left(m_{v} h_{0, e v o}+m_{l} h_{0, e l o}\right)=W_{c o m p}
$$

where $W_{\text {comp }}$ is the input power of the compressor; the total discharge enthalpy of compressor $h_{0, c v o}$ is given in Equation (8).

$$
h_{0, c v o}=h_{c v o}+\frac{u_{c v o}^{2}}{2 \times 1000}
$$

The vapor enthalpy at the compressor outlet $h_{c v o}$ is determined by the discharge temperature and pressure. According to Equation (7), given the discharge enthalpy and input power of the compressor, the amount of liquid entrainment $m_{l}$ can be calculated and, conversely, if it is assumed that there is no liquid drawn into the compressor, the discharge enthalpy and temperature of the compressor without liquid entrainment can be calculated using Equation (7). So, the difference between the measured compressor discharge temperature and the calculated value without liquid carryover reflects the amount of liquid entrainment.

The input power of the compressor in Equation (7) is calculated by Equation (9) according to the motor efficiency and the transmission power loss.

$$
W_{\text {comp }}=W_{\text {in }} \eta_{e}-Q_{m e c h}
$$

By adding Equations (1) and (7) and using Equations (5), (6) and (9), the following equation can be obtained:

$$
\left(m_{v}+m_{l}\right)\left(h_{0, c v o}-h_{\text {sub }}\right)=Q_{e}+W_{\text {in }}
$$


The energy balance of the condenser can be written as:

$$
Q_{e}+W_{i n}=Q_{c}
$$

Therefore, Equation (10) can also be expressed as Equation (12).

$$
\left(m_{v}+m_{l}\right)\left(h_{0, c v o}-h_{\text {sub }}\right)=Q_{c}
$$

The condenser heat exchange capacity $Q_{c}$ is calculated by Equation (13).

$$
Q_{c}=\rho_{c w} V_{c w} c_{c w}\left(t_{c w o}-t_{c w i}\right)-V_{c w} \Delta p_{c w}
$$

According to Equations (7) and (10) and by using Equation (9), $m_{l}$ can be obtained by the following equation:

$$
m_{l}=\frac{W_{\text {in }} \eta_{e}-Q_{m e c h}}{h_{0, e v o}-h_{0, e l o}}-\frac{\left(Q_{e}+W_{i n}\right)\left(h_{0, \text { cvo }}-h_{0, e v o}\right)}{\left(h_{0, e v o}-h_{0, e l o}\right)\left(h_{0, c v o}-h_{\text {sub }}\right)}
$$

The above equation uses the heat exchange rate of the evaporator and the electric power input of the compressor to calculate the liquid entrainment rate $m_{l}$. By using Equation (11), another expression for calculating the amount of liquid entrainment can be obtained, as described in Equation (15).

$$
m_{l}=\frac{W_{i n} \eta_{e}-Q_{\text {mech }}}{h_{0, \text { evo }}-h_{0, e l o}}-\frac{\left(Q_{e}+W_{i n}+Q_{c}\right)\left(h_{0, c v o}-h_{0, e v o}\right)}{2\left(h_{0, e v o}-h_{0, e l o}\right)\left(h_{0, c v o}-h_{\text {sub }}\right)}
$$

Since Equation (15) uses both heat exchange capacities of evaporator and condenser to calculate the liquid entrainment rate, generally, the result from Equation (15) has higher accuracy than that from Equation (14). If the system is in complete thermal balance, the results from Equations (14) and (15) are the same.

The thermal balance of a chiller system is defined by Equation (16).

$$
T B=\left(\frac{Q_{e}+W_{i n}}{Q_{c}}-1\right) \times 100 \%
$$

After obtaining $m_{l}$ according to Equation (14) or Equation (15), one can further obtain $m_{v}$ using Equation (10), then the entrainment factor $E_{f g}$ can be obtained by Equation (17).

$$
E_{f g}=\frac{m_{l}}{m_{v}}
$$

To calculate the entrainment factor according to Equations (1)-(17), it is necessary to measure the cooling capacity of the evaporator, the heat dissipation rate of the condenser, the input power and the efficiency of the motor, the transmission power loss, the outlet state of the condenser and the suction and discharge states of the compressor. The motor efficiency and the transmission power loss are calculated by the fitting correlations of the data provided by the manufacturer. The motor efficiency is calculated by cubic curve fitting based on the efficiency data provided by the manufacturer at $25 \%, 50 \%, 75 \%, 100 \%$ and $115 \%$ load, as shown in Equation (18).

$$
\eta_{e}=0.03499 \times \text { Load }^{3}-0.1028 \times \text { Load }^{2}+0.07993 \times \text { Load }+0.9489
$$

where Load is the load factor of the motor. The motor efficiency during the experiments is around $96.5 \%$. The load factor of the experimental points is between $67-90 \%$, which is within the range of fitting data. Another way to calculate the motor efficiency is to use a linear interpolation method between a series of given points [18]. Within the scope of the experiment, the difference in motor efficiency calculated by the two methods is less than $0.1 \%$. 
The transmission power loss $Q_{\text {mech }}$ includes gear mesh loss and the related bearing loss. For a constant speed chiller, $Q_{\text {mech }}$ is obtained by linear fitting according to the transmission loss data provided by the manufacturer at the maximum and minimum motor output power, given in Equation (19).

$$
Q_{\text {mech }}=0.00381 \times\left(W_{i n} \eta_{e}\right)+14.03
$$

The transmission efficiency $\eta_{\text {mech }}$ during the experiments is $3-4 \%, \eta_{\text {mech }}$ is defined by Equation (20).

$$
\eta_{\text {mech }}=Q_{\text {mech }} /\left(W_{\text {in }} \eta_{e}\right)
$$

\subsection{Experimental Measurement}

\subsubsection{Test Chiller Unit}

To verify the above measurement method, a single-stage water-cooled centrifugal chiller with a cooling capacity of 700 U.S. RT manufactured by a local supplier was tested. The refrigerant used is R134a. The chiller consists of a single-stage centrifugal compressor, a shell-and-tube condenser, a flooded evaporator, an orifice plate and a motor and a transmission system. There is a subcooling tube bundle at the bottom of the condenser and the condenser outlet has a certain degree of subcooling under the test conditions, which meets the conditions of the Equation (1) above. The compressor is a constant speed compressor with a rated cooling capacity of $940 \mathrm{RT}$ and is intentionally oversized to measure liquid carryover performance.

The flooded evaporator tested has an inner diameter of $800 \mathrm{~mm}$. It holds 535 tubes with an outer diameter of $18.85 \mathrm{~mm}$ and a length of $3.92 \mathrm{~m}$. The tubes are in a rotated triangle arrangement and the pitch to diameter ratio is 1.167 . The headspace above the bundle is $366.1 \mathrm{~mm}$ high and the inner diameter of the top outlet is $309 \mathrm{~mm}$. There is no baffle structure in the headspace. The tube side of the evaporator is a bottom-to-top two-tube-pass arrangement.

\subsubsection{Instrumentation}

Figure 3 shows the experimental measuring points of the refrigeration system. The suction and discharge pipes of the compressor are both a 90-degree elbow, which connects the compressor and the evaporator and the compressor and the condenser respectively. The discharge temperature and pressure measurement points are located on the discharge elbow, close to the condenser end. The discharge temperature measuring point locates at the center of the elbow and the pressure measuring point locates at the horizontal position of the elbow circumference. The suction temperature and pressure points are located on the suction elbow, near the evaporator end. The suction temperature measuring point locates at the center of the elbow and the pressure measuring point locates at the horizontal position of the elbow circumference.

All instruments were calibrated before the experiment. Table 1 lists the instrument and uncertainty of the measured parameters. Since a water mixer was installed before the inlet and outlet temperature measurement points of the evaporator and condenser, respectively, it can be considered that the inlet and outlet water temperatures of the evaporator and condenser are uniform and the measurement uncertainty of the water temperature depends on the sensor accuracy. Considering the possible non-uniform distribution of suction and discharge temperatures on the cross-section of measuring points, the uncertainty of both suction and discharge temperatures is assumed to be $\pm 0.1^{\circ} \mathrm{C}$ (the influence of measurement uncertainty will be analyzed below). All parameters are collected by an Agilent data logger. Then, the data are read, processed and stored using a program running in Labpro. 


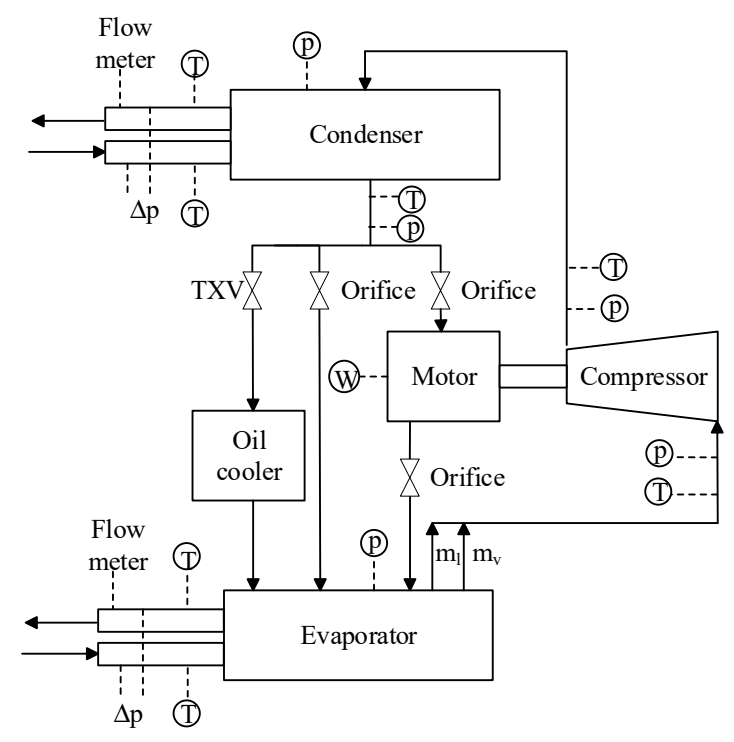

Figure 3. Arrangement of measuring points for liquid entrainment of a centrifugal chiller.

Table 1. Measurement parameters and uncertainties.

\begin{tabular}{ccc}
\hline Parameter & Sensor Type & Uncertainty \\
\hline Water temperature & Pt-100 resistance (water) & $\pm 0.05 \mathrm{~K}$ \\
Refrigerant temperature & Pt-100 resistance (refrigerant) & $\pm 0.1 \mathrm{~K}$ \\
Refrigerant Pressure at low pressure side of the unit & Pressure piezoelectric transducer & $\pm 1.4 \mathrm{kPa}( \pm 0.2 \% \mathrm{FS})$ \\
Refrigerant pressure at high pressure side of the unit & Pressure piezoelectric transducer & $\pm 3.2 \mathrm{kPa}( \pm 0.2 \% \mathrm{FS})$ \\
Water volumetric flow rate & Electromagnetic flowmeter & $\pm 2.4 \mathrm{~m}^{3} \cdot \mathrm{h}^{-1}( \pm 0.3 \% \mathrm{FS})$ \\
Evaporator, condenser water pressure drop & Capacitive differential pressure transducer & $\pm 3 \mathrm{kPa}( \pm 0.5 \% \mathrm{FS})$ \\
Electric power input & Digital Powermeter & $\pm 2.6 \mathrm{~kW}( \pm 0.2 \%)$ \\
\hline
\end{tabular}

Based on vendor data, the uncertainty of motor efficiency is $0.5 \%$ and the uncertainty of transmission power loss is set at $15 \%$.

\subsubsection{Content and Procedure}

The experiment was carried out under AHRI full load conditions [16]. Under a certain fixed cooling capacity, the performance of the chiller was measured with increasing refrigerant charge and the variations of $\mathrm{COP}$, evaporation temperature, condensation temperature and discharge temperature with refrigerant charge were obtained. The entrainment factor $E_{f g}$ was calculated based on Equations (14) and (15). A total of three cooling capacities, i.e., 600,700 and 800 RT, were tested. The cooling capacity of the chiller during the charging process was kept basically unchanged by adjusting the opening of the inlet guide vane of the compressor.

The experiment started with a refrigerant charge of about $519 \mathrm{~kg}$. At each fixed cooling capacity, the charge was increased by about $15 \mathrm{~kg}$ each time. The adjustment method of working condition is as follows: adjust the pump speed of cold water and cooling water, respectively, to make the flow of cold water and cooling water reach the set value; adjust the flow of the cooling water loop into the cold water loop through a regulating valve to make the outlet temperature of cold water reaches the set value; adjust the flow of cooling tower water into the cooling water loop through a regulating valve to make the inlet temperature of cooling water reach the set value.

Data acquisition was performed after the working conditions are stable. The time interval is about $20 \mathrm{~min}$ and the average value in each time period is taken as the measured value. To ensure stability, during the data acquisition period, the temperature change at the inlet and outlet of the evaporator and condenser was less than $0.2{ }^{\circ} \mathrm{C}$ and the water volumetric flow rate change was less than $1 \%$. The span of the refrigerant charge during 
the study is $519-612 \mathrm{~kg}$ and the range is large enough so that the evaporation temperature of the evaporator at each cooling capacity tends to be constant finally.

Figures 4 and 5 show the raw data of inlet and outlet water temperature of evaporator and condenser under the condition of $700 \mathrm{RT}$ and $581 \mathrm{~kg}$ charge (the full charge for the evaporator), respectively. It can be seen from the figures that when the working condition reaches a stable state, the inlet and outlet water temperatures of evaporator and condenser change very slightly, less than $0.05^{\circ} \mathrm{C}$. Similar results were obtained for other conditions.

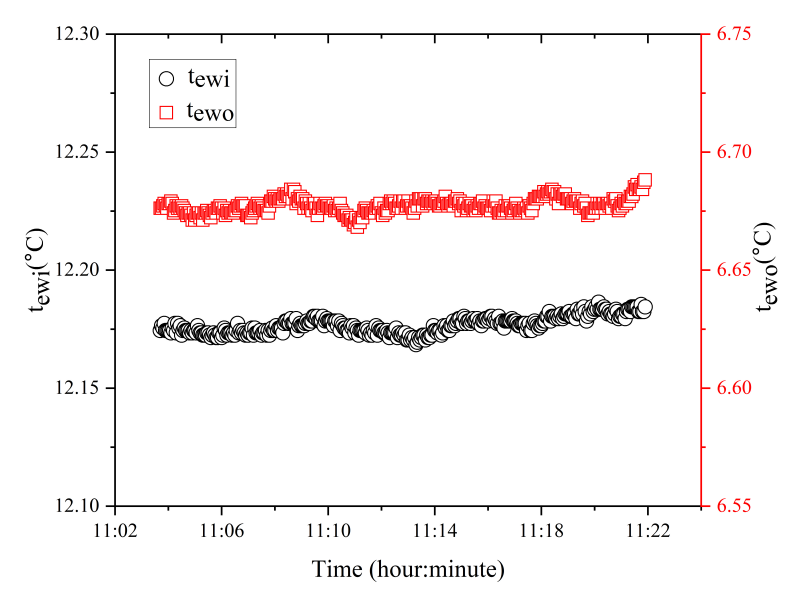

Figure 4. Evaporator inlet and outlet water temperature at $700 \mathrm{RT}$ and $581 \mathrm{~kg}$ charge.

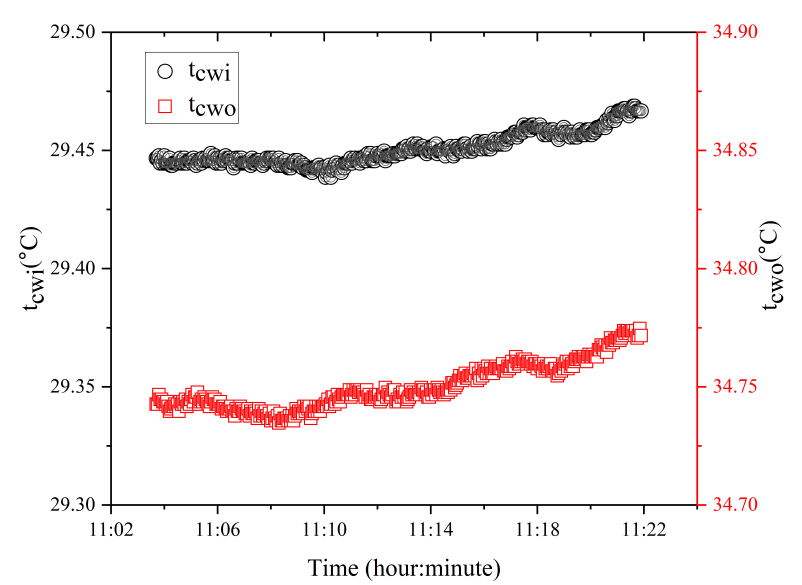

Figure 5. Condenser inlet and outlet water temperature at $700 \mathrm{RT}$ and $581 \mathrm{~kg}$ charge.

\subsection{Uncertainty Analysis}

The uncertainty of $E_{f g}$ is calculated by the uncertainty propagation equation according to the uncertainty of each parameter [19]. Taking Equation (14) as an example, the uncertainty of $E_{f g}$ is calculated by the following Equation (21).

$$
\begin{aligned}
& u\left(E_{f g}\right)=\sqrt{\left(\frac{\partial E_{f g}}{\partial W_{i n}}\right)^{2}\left(u\left(W_{i n}\right)\right)^{2}+\left(\frac{\partial E_{f g}}{\partial \eta_{e}}\right)^{2}\left(u\left(\eta_{e}\right)\right)^{2}+\left(\frac{\partial E_{f g}}{\partial Q_{\text {mech }}}\right)^{2}\left(u\left(Q_{\text {mech }}\right)\right)^{2}+\left(\frac{\partial E_{f g}}{\partial V_{e w}}\right)^{2}\left(u\left(V_{e w}\right)\right)^{2}} \\
& \overline{+\left(\frac{\partial E_{f g}}{\partial t_{e w i}}\right)^{2}\left(u\left(t_{\text {ewi }}\right)\right)^{2}+\left(\frac{\partial E_{f g}}{\partial t_{e w o}}\right)^{2}\left(u\left(t_{\text {ewo }}\right)\right)^{2}+\left(\frac{\partial E_{f g}}{\partial t_{s u c t}}\right)^{2}\left(u\left(t_{\text {suct }}\right)\right)^{2}+\left(\frac{\partial E_{f g}}{\partial t_{d i s}}\right)^{2}\left(u\left(t_{\text {dis }}\right)\right)^{2}} \\
& \overline{+\left(\frac{\partial E_{f g}}{\partial t_{l o}}\right)^{2}\left(u\left(t_{l o}\right)\right)^{2}+\left(\frac{\partial E_{f g}}{\partial p_{s u c t}}\right)^{2}\left(u\left(p_{\text {suct }}\right)\right)^{2}+\left(\frac{\partial E_{f g}}{\partial p_{d i s}}\right)^{2}\left(u\left(p_{\text {dis }}\right)\right)^{2}+\left(\frac{\partial E_{f g}}{\partial p_{c}}\right)^{2}\left(u\left(p_{c}\right)\right)^{2}} \\
& \overline{+\left(\frac{\partial E_{f g}}{\partial \Delta p_{e}}\right)^{2}\left(u\left(\Delta p_{e}\right)\right)^{2}}
\end{aligned}
$$

The experimental data processing and uncertainty calculation are all based on the Engineering Equation Solver (EES) [20]. The thermal properties of refrigerant and water are calculated by the EES built-in program. The authors examined the accuracy of the 
EES thermal property calculations and compared the difference between the $E_{f g}$ calculated using Refprop 9.1 (Gaithersburg, USA) [21] and the results from EES. It was found that the difference is generally much less than $0.1 \%$. So, it is reasonable to ignore the EES thermal property calculation error.

Table 2 shows the entrainment factor $E_{f g}$ and its uncertainty under 700 RT and $581 \mathrm{~kg}$ of charge, including the results using Equation (14) (Method 1) and Equation (15) (Method 2 ). The table also shows the impact on the $E_{f g}$ results if the uncertainty of the transmission power loss is doubled. As shown in the table, the calculated $E_{f g}$ value of Equation (15) is about $2 \%$ lower than that of Equation (14). The difference is related to the system thermal balance, the better the system thermal balance, the smaller the difference between the two methods will be. Since the thermal balance of this experimental point is $-0.23 \%$ (the thermal balance of all test points is within $-0.80 \% \sim-0.18 \%$ ), the difference between the two methods is small. The thermal balance of the system can partly reflect the reliability of the measurement results, so it is recommended that the thermal balance of the experimental points be within $1 \%$. As shown in the table, the relative uncertainties of $E_{f g}$ for methods one and two are $24 \%$ and $22 \%$, respectively. Since the results from the two methods are very close to each other for a system with good thermal balance and the accuracy of Method 2 is higher, it is recommended to use Method 2 (Equation (15)) to calculate $E_{f g}$. As can be seen from the table, if the uncertainty of the transmission power loss is doubled, the relative uncertainty of the $E_{f g}$ of methods one and two will increase to $29 \%$ and $27 \%$, respectively. Therefore, the transmission power loss has a great impact on the uncertainty of $E_{f g}$. The accuracy of the transmission power loss can be improved by directly measuring the heat exchange rate of the oil cooler.

Table 2. $E_{f g}$ and its uncertainty at $700 \mathrm{RT}$ and $581 \mathrm{~kg}$ charge.

\begin{tabular}{ccc}
\hline & \multicolumn{2}{c}{$E_{f g}$} \\
\cline { 2 - 3 } & Baseline & Double Uncertainty of $Q_{\text {mech }}$ \\
\hline Method 1-Equation (14) & $0.91 \% \pm 0.22 \%$ & $0.91 \% \pm 0.26 \%$ \\
Method 2-Equation (15) & $0.89 \% \pm 0.20 \%$ & $0.89 \% \pm 0.24 \%$ \\
\hline
\end{tabular}

Table 3 shows the uncertainty of the input parameters under this condition and their contribution to the uncertainty of the entrainment factor $E_{f g}$. The input parameters include the measured parameters / those provided according to the manufacturer. From Table 3, it can be seen that for Method 2 using Equation (15), the uncertainty of $E_{f g}$ depends mainly on the uncertainty of the inlet and outlet water temperature of the evaporator and condenser, motor input power, motor efficiency, transmission power loss, suction and discharge temperature. Other parameters, including the water flow rate of the evaporator and condenser, suction and discharge pressure, condenser outlet temperature and the water pressure drop of the evaporator and condenser, have little effect on $E_{f g}$ uncertainty (an effect of $\pm 1 \%$ or less on the relative uncertainty). For Method 1, using Equation (14), since the heat exchange capacity of the condenser is not involved, the inlet and outlet water temperature and the water flow rate of the evaporator have greater influences on the uncertainty of $E_{f g}$, but the impacts of other parameters are relatively less.

Table 3. Uncertainty of input parameters and their contributions to $E_{f g}$ uncertainty.

\begin{tabular}{|c|c|c|c|c|c|c|c|c|c|c|c|c|c|c|c|c|}
\hline & $t_{e w i}$ & $t_{\text {ewo }}$ & $V_{e w}$ & $t_{c w i}$ & $t_{c w o}$ & $V_{c w}$ & $W_{\text {in }}$ & $\eta_{e}$ & $p_{\text {suct }}$ & $t_{\text {suct }}$ & $p_{\text {dis }}$ & $t_{d i s}$ & $t_{l o}$ & $Q_{m e c h}$ & $\Delta p_{e w}$ & $\Delta p_{c w}$ \\
\hline Unit & ${ }^{\circ} \mathrm{C}$ & ${ }^{\circ} \mathrm{C}$ & $\mathrm{m}^{3} \cdot \mathrm{s}^{-1}$ & ${ }^{\circ} \mathrm{C}$ & ${ }^{\circ} \mathrm{C}$ & $\mathrm{m}^{3} \cdot \mathrm{s}^{-1}$ & $\mathbf{k W}$ & $\%$ & $\mathbf{k P a}$ & ${ }^{\circ} \mathrm{C}$ & $\mathbf{k P a}$ & ${ }^{\circ} \mathrm{C}$ & ${ }^{\circ} \mathrm{C}$ & $\mathrm{kW}$ & $\mathbf{k P a}$ & $\mathbf{k P a}$ \\
\hline Data & 12.18 & 6.68 & 0.1058 & 34.75 & 29.45 & 0.1324 & 455.7 & $96.5 \%$ & 361.2 & 6.0 & 904.0 & 44.3 & 32.9 & 15.7 & 63.7 & 62.1 \\
\hline Uncertainty & 0.05 & 0.05 & $6.7 \times 10^{-4}$ & 0.05 & 0.05 & $6.7 \times 10^{-4}$ & 2.6 & $0.5 \%$ & 1.4 & 0.1 & 3.2 & 0.1 & 0.1 & 2.4 & 3.0 & 3.0 \\
\hline $\begin{array}{l}\text { of uncertainty- } \\
\text { Equation (15) }\end{array}$ & $6.9 \%$ & $7.0 \%$ & $3.4 \%$ & $10.7 \%$ & $10.7 \%$ & $3.0 \%$ & $14.4 \%$ & $13.9 \%$ & $1.0 \%$ & $5.8 \%$ & $2.5 \%$ & $5.7 \%$ & $0.3 \%$ & $14.8 \%$ & $0.0 \%$ & $0.0 \%$ \\
\hline $\begin{array}{l}\text { of uncertainty- } \\
\text { Equation (14) }\end{array}$ & $22.2 \%$ & $22.3 \%$ & $10.8 \%$ & & & & $9.7 \%$ & $11.1 \%$ & $0.8 \%$ & $4.6 \%$ & $2.0 \%$ & $4.5 \%$ & $0.3 \%$ & $11.8 \%$ & $0.0 \%$ & \\
\hline
\end{tabular}




\section{Results and Discussion}

The liquid entrainment of the evaporator is related to the cooling capacity, chiller operating conditions and the liquid level in the evaporator. Under fixed cooling capacity and operating conditions, with the increase of the refrigerant charge, the liquid level in the evaporator rises and the number of tubes participating in boiling heat transfer gradually increases, which results in the evaporation temperature gradually increasing and the heat transfer temperature difference between cold water and refrigerant gradually decreasing, i.e., the total heat transfer coefficient (HTC), $K$, of the evaporator gradually increases. This process continues until the bundle is fully immersed in liquid refrigerant, at this time the evaporative temperature increases to the highest and the total HTC reaches the maximum. Therefore, under fixed cooling capacity and operating conditions, $K$ of the evaporator can be used to indirectly reflect the change of the evaporator liquid level with the charge, as shown in Figure 6. The total HTC of the evaporator is calculated by the following Equation (22).

$$
K=\frac{Q_{e}}{A_{e} \Delta t}
$$

where $A_{e}$ is the total heat transfer area of the evaporator based on the envelope diameter of the enhanced tube and $\Delta t$ is the logarithmic mean temperature difference between the refrigerant and cold water, calculated by Equation (23).

$$
\Delta t=\frac{t_{e w i}-t_{e w o}}{\ln \left(\frac{t_{e w i}-t_{e}}{t_{e w 0}-t_{e}}\right)}
$$

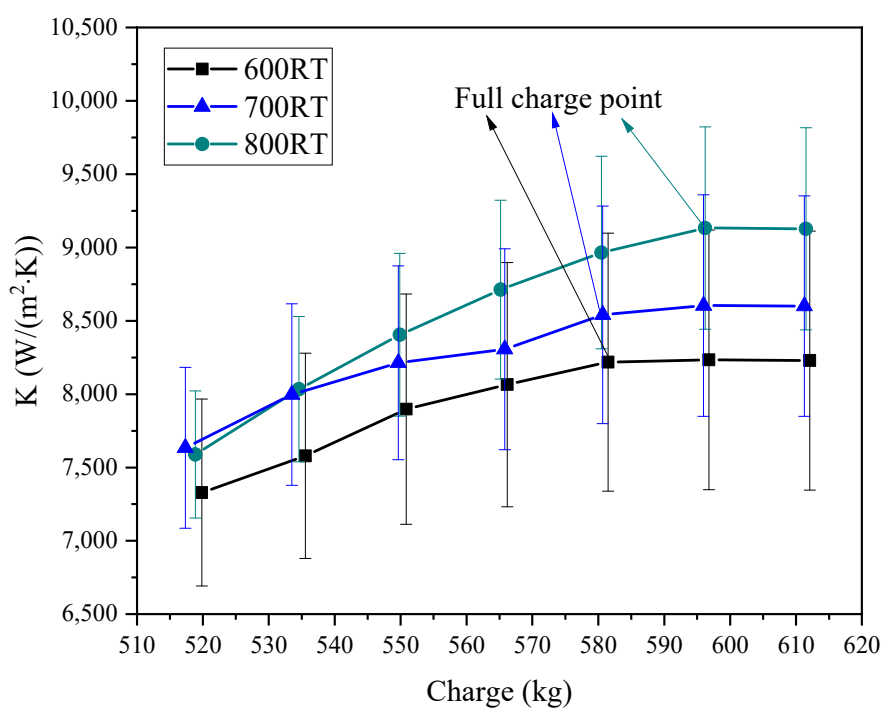

Figure 6. Evaporator total HTC as a function of charge at each cooling capacity.

It can be seen from Figure 6 that under fixed operating conditions for each cooling capacity, K gradually increases with the increase of charge. At 600 and 700 RT, when the refrigerant charge is about $581 \mathrm{~kg}, K$ reaches the maximum value. The amount of refrigerant continues to increase subsequently; however, there is little variation of $K$. At $800 \mathrm{RT}$, when the refrigerant charge is $596 \mathrm{~kg}, K$ reaches the maximum, indicating that as the cooling capacity increases, more refrigerant is needed so that the evaporator tube bundle can be fully immersed. It can be seen from Figure 6 that $K$ increases by about $12 \%, 12 \%$ and $20 \%$ respectively during the charging process at 600,700 and 800 RT.

Figure 7 shows the variation of entrainment factor with charge under 700 RT AHRI conditions, where $E_{f g}-1$ and $E_{f g}-2$ represent $E_{f g}$ calculated by Equation (14) (Method 1) and Equation (15) (Method 2), respectively. It can be seen that at this cooling capacity, the variation of $E_{f g}$ with charge conforms to a quadratic curve: with the increase of charge, $E_{f g}$ gradually increases and the growth rate gradually accelerates. This is because, on the one 
hand, with the increase of charge, the liquid level in the evaporator rises. Under the shear entrainment of the vapor flow inside the tube bundle, liquid droplets can enter a higher position in the headspace above the bundle with a certain initial velocity, making it easier to be drawn into the compressor to form the liquid carryover. On the other hand, when the liquid level exceeds the bundle, a layer of liquid will be formed on the bundle, which reduces the height of the space above the bundle and the wave action of the liquid layer will entrain more large droplets into the headspace, further increasing the amount of liquid entrainment [13]. The reason for the close calculation results of the two methods is that the thermal balance at each experimental point is within $1 \%$ as mentioned before.

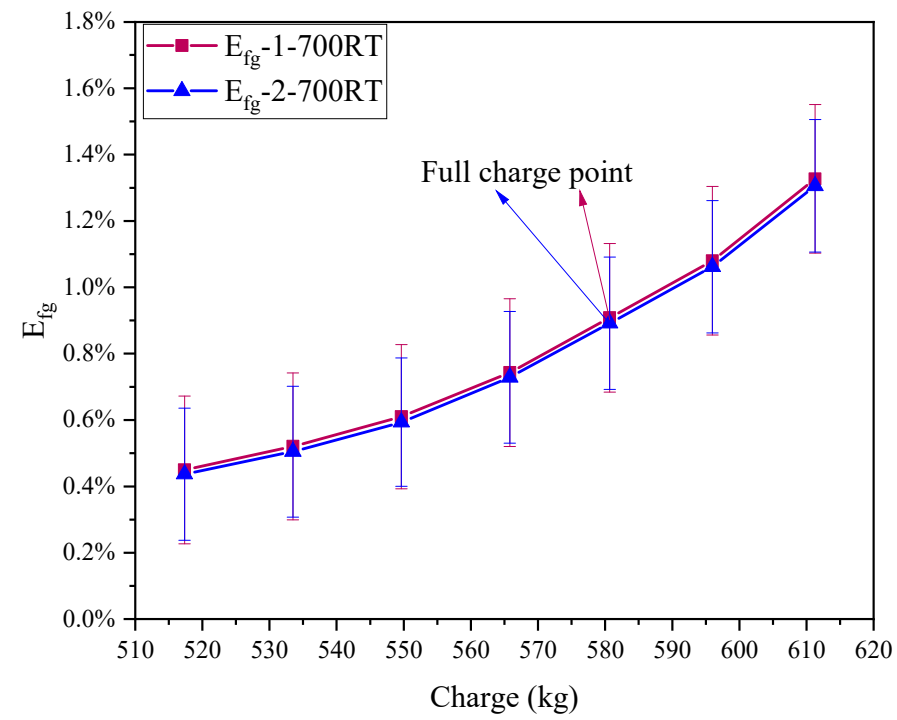

Figure 7. Liquid entrainment factor as a function of charge at 700 RT.

Figure 7 also shows the uncertainty of each experimental point. It can be seen from the figure that the uncertainty of $E_{f g}$ remains essentially unchanged as charge increases and the average uncertainties of the two methods are $0.22 \%$ and $0.20 \%$, respectively. Because $E_{f g}$ is small at low charge, the relative uncertainty at point 1 is large, reaching about $50 \%$. However, for the evaporator carryover problem of a centrifugal chiller, the meaningful work condition is the one with large cooling capacity and with tube bundle full immersed in liquid refrigerant, which corresponds to the experimental point of $581 \mathrm{~kg}$ charge in Figure 7 (see Figure 6 above), where the relative uncertainties of methods one and two are $24 \%$ and $22 \%$, respectively (as shown in Table 2 ). Due to the relatively small experimental uncertainty of Method 2, only the results from Method 2 are shown later in this paper.

Figure 8 shows the variation of the entrainment factor with charge under different cooling capacities and AHRI full load conditions. As a comparison, the results at 700 RT are also shown in this figure. It can be seen that the entrainment factor is small at $600 \mathrm{RT}$ and increases smoothly with the increase of charge. However, the variation trend at 800 RT condition is similar to that at $700 \mathrm{RT}$, with the entrainment factor increasing slowly at the beginning and gradually accelerating later. Since the cooling capacity relates to the vapor superficial velocity $j_{v}$ in the vertical direction and the refrigerant charge value relates to the space height $h$ above the liquid surface, the relationship between the liquid entrainment factor $E_{f g}$ and the charge amount under different cooling capacities in Figure 8 actually reflects the dependence of $E_{f g}$ on $j_{v} / h$. Similar to the pool entrainment in the momentum control region, the dependence of the entrainment factor on $j_{v} / h$ varies with the vapor velocity (cooling capacity) [8]. Due to lower cooling capacity, the two-phase flow pattern in the tube bundle at 600 RT may be different from that at 700 and 800 RT and the initial velocity and diameter of droplets generated are different, resulting in different entrainment factor dependence with $j_{v} / h$, that is, the relationship between $E_{f g}$ and refrigerant charge is 
different under different cooling capacity. Figure 8 also shows that the average uncertainties of $E_{f g}$ at 600 and $800 \mathrm{RT}$ are $0.21 \%$ and $0.18 \%$, respectively.

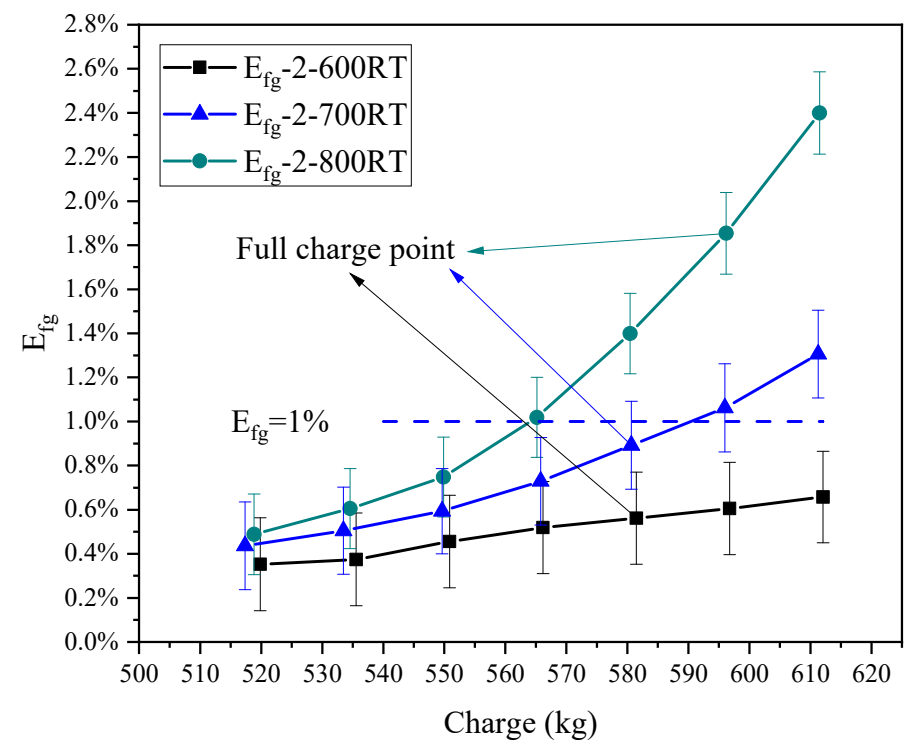

Figure 8. Liquid entrainment factor as a function of charge at each cooling capacity.

It can be seen from Figure 8 that under the full charge of the evaporator (point 5 on the 600 RT and 700 RT curves and point 6 on the 800 RT curve), the $E_{f g}$ values at 600,700 and $800 \mathrm{RT}$ are $0.56 \%, 0.89 \%$ and $1.85 \%$, respectively, and $E_{f g}$ increases rapidly with the increase of cooling capacity. If it is required that the reasonable $E_{f g}$ is no more than $1 \%$ when the unit is running, the refrigerant charge at $800 \mathrm{RT}$ must be reduced to less than $565 \mathrm{~kg}$, which is less than $596 \mathrm{~kg}$ of refrigerant required for the evaporator tube bundle to be fully immersed in liquid refrigerant at this working condition. This means that there are "dry tubes" in the evaporator when the unit is running, resulting in about $5 \%$ of the heat transfer performance loss at 800 RT, as shown in Figure 6. By using $E_{f g}=1 \%$ as the limit entrainment factor, the reasonable maximum cooling capacity of the evaporator limited by the liquid entrainment under AHRI conditions is between 700 and 800 RT.

The variation of system COP is caused by the changes in system cooling capacity, system temperature lift (condensation temperature-evaporation temperature, LIFT) and entrainment factor $E_{f g}$. As LIFT increases, COP decreases. Compared with the rated cooling capacity of the compressor in the experiment, the tested capacities of 600, 700 and 800 RT are all part loads. If the LIFT remains unchanged, the system COP increases with the increase in cooling capacity. Figure 9 shows the variation of evaporation temperature $t_{e}$ and the temperature LIFT with charge at each cooling capacity. It can be seen from the figure that as the charge increases, the evaporation temperature at each cooling capacity increases gradually and then remains almost unchanged after the tube bundle is fully immersed in the refrigerant. Figure 9 also shows that with the increase of the charge, the LIFT experimental value is almost unchanged at $600 \mathrm{RT}$ and the LIFTs of the system are reduced by $0.11^{\circ} \mathrm{C}$ and $0.18{ }^{\circ} \mathrm{C}$ at 700 and $800 \mathrm{RT}$, respectively. 


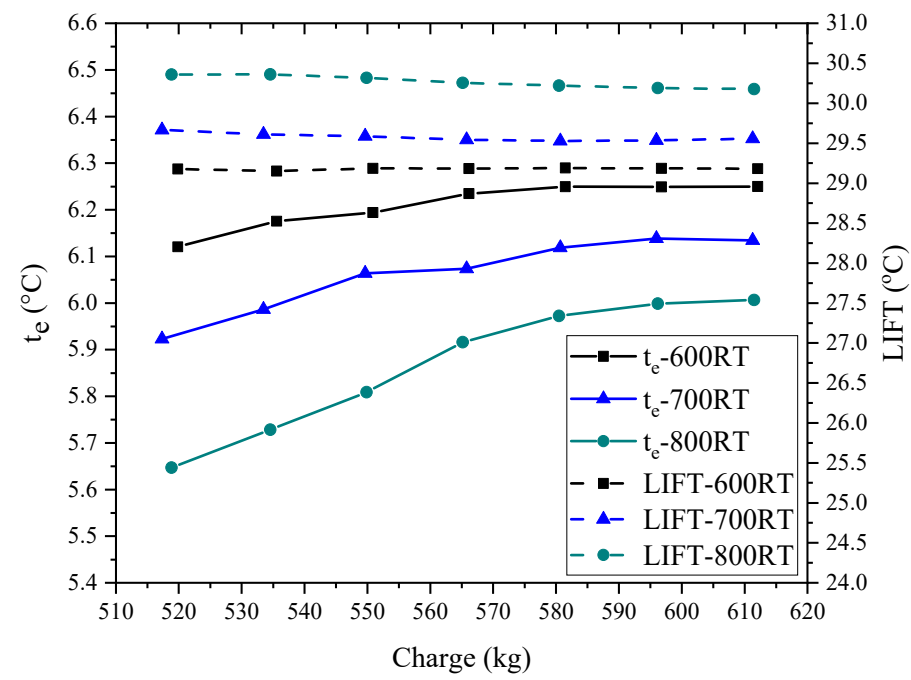

Figure 9. $t_{e}$, LIFT as a function of charge at each cooling capacity.

The influence of the variation of the cooling capacity, evaporation temperature and condensation temperature on COP can be considered by the calculation based on the compressor efficiency map, which is obtained at a certain suction superheat, so it can be considered that there is no liquid carryover effect. Therefore, the difference between the calculated and test COP can reflect the effect of the liquid entrainment factor. Figure 10 shows the efficiency factor contours of the test compressor, where $\Phi$ is the percent of the rated volumetric flow rate and $\Psi$ is the percent of the rated polytropic work, as described by Equations (24) and (26), respectively.

$$
\Phi=\frac{V_{s}}{V_{n}}
$$

where $V_{n}$ is the rated volumetric flow rate, $V_{n}=1.283 \mathrm{~m}^{3} \cdot \mathrm{s}^{-1} . V_{s}$ is the refrigerant volumetric flow rate at evaporator state, calculated by Equation (25).

$$
V_{s}=m_{r} v_{e}
$$

where $v_{e}$ is the specific volume of refrigerant vapor at evaporator state, determined by the evaporation pressure and the suction enthalpy. $m_{r}$ is the refrigerant flow rate calculated by Equation (1) assuming no liquid carryover.

$$
\Psi=f \ln \left(p_{c} / p_{e}\right) \frac{p_{c} v_{c}-p_{e} v_{e}}{\ln \left(p_{c} v_{c} / p_{e} v_{e}\right)} / w_{n}
$$

where $p_{c}, p_{e}$ are condensation and evaporation pressure respectively, $v_{c}$ is the specific volume of refrigerant vapor at condenser state, determined by the condensation pressure and discharge enthalpy; $f$ correction factor is introduced to correct the error of calculating the polytropic compression work by using the approximate polytropic process equation [22] $f$ is about 1.005 for all test points. $w_{n}$ is the rated specific polytropic work, $w_{n}=21.4 \mathrm{~kJ} \cdot \mathrm{kg}^{-1}$. Once $\Phi$ and $\Psi$ are known, the efficiency factor $\eta$ of the compressor is obtained from the compressor map, then the polytopic efficiency $\eta_{c}$ is calculated by Equation (27).

$$
\eta_{c}=\eta \eta_{n}
$$

where $\eta_{n}$ is the rated compressor efficiency, $\eta_{n}=0.802$, provided by the chiller manufacturer. By adding the polytropic compression power, transmission loss and motor loss, the input power of the chiller can be obtained, thereby obtaining the calculated COP. 


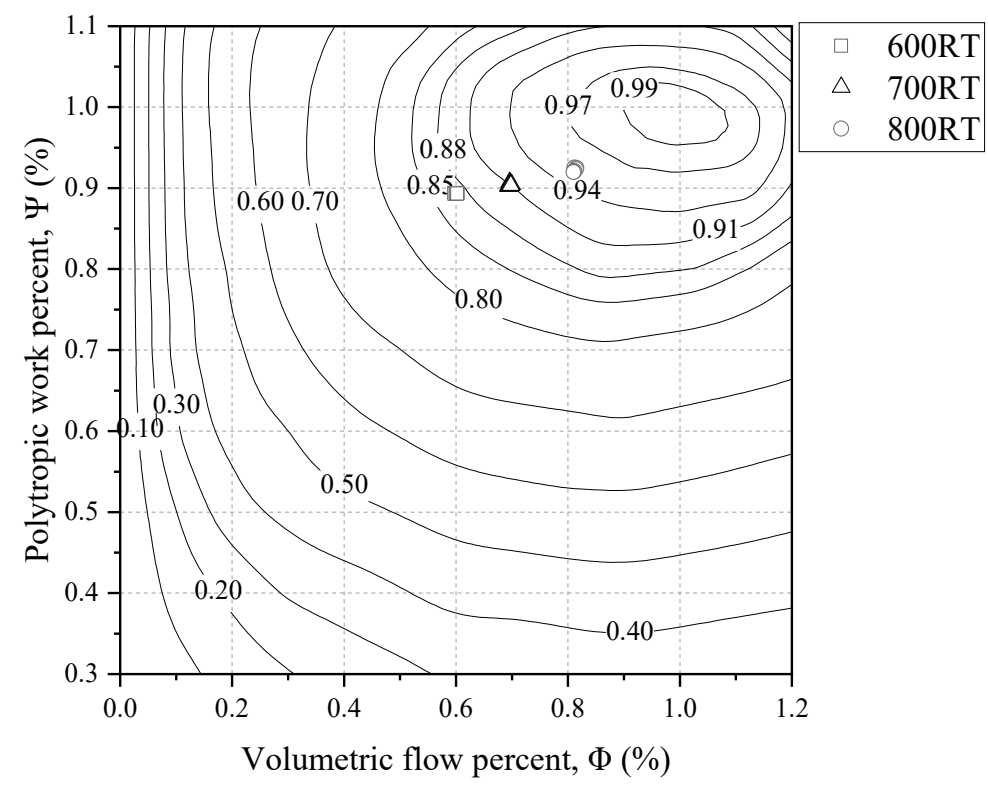

Figure 10. Compressor efficiency factor map.

Figure 10 also shows each test point. It can be seen from the figure that the test points coincide under each cooling capacity. The variation of $\Phi$ and $\Psi$ under each cooling capacity is less than $1.0 \%$ and the variation of efficiency factor is less than $0.5 \%$. It can also be seen from the figure that if LIFT ( $\Psi$ ) remains unchanged, the compressor efficiency factor increases with the increase of cooling capacity under part load.

Figure 11 shows the variation of test and calculated COP with refrigerant charge at each cooling capacity. The calculated COP values of each curve in Figure 11 were corrected by a constant $(0.06,-0.03,-0.05$ for $600,700,800 \mathrm{RT}$, respectively) so that the calculated $\mathrm{COP}$ at the first point of each curve coincides with the experimental value, to eliminate the influence of the calculation model error and clearly reflect the trend of the influence of the liquid entrainment on COP. As can be seen from Figure 11, the variation of the calculated COP is small (less than $0.5 \%$ ) for each curve, mainly because the temperature LIFT is almost constant for each curve $\left(0-0.18{ }^{\circ} \mathrm{C}\right.$, see Figure 9$)$ and the variations of the cooling capacity are also small for each curve (1.1-1.4\%).

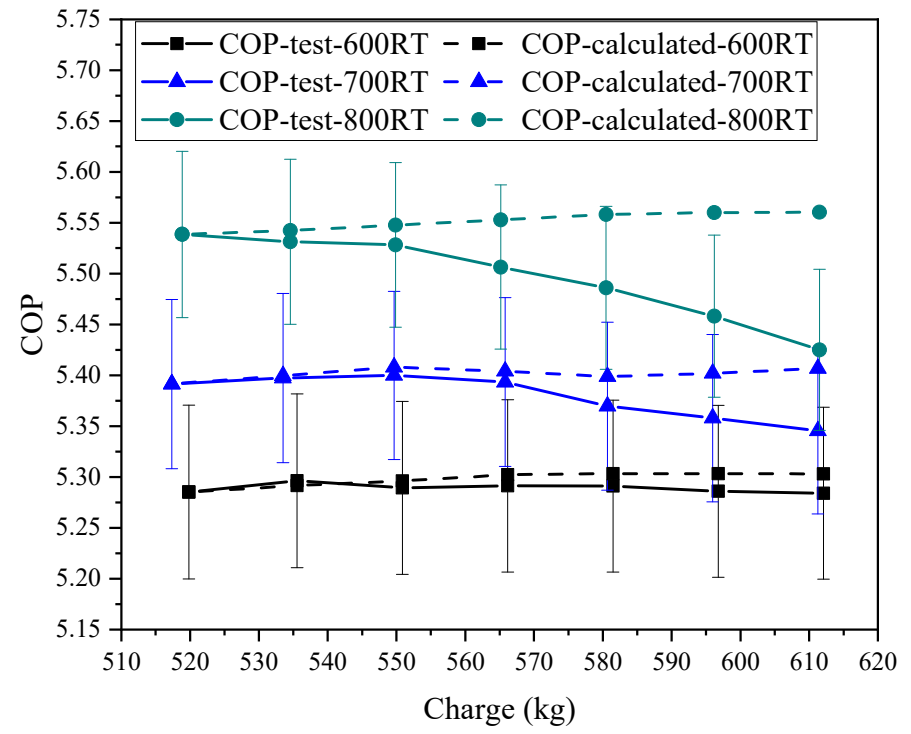

Figure 11. Test and calculated COP as a function of charge at each capacity. 
For the test chiller, the most important factor that causes the variation of COP is the $E_{f g}$ and its variation. If the variation of cooling capacity and LIFT is not considered, the system COP decreases with the increase of $E_{f g}$, because the liquid droplets entrained by the vapor do not contribute to the corresponding cooling capacity, while the evaporation and compression of the liquid droplets in the compressor consumes compression power. Moreover, due to the density difference under vapor and liquid phases and the interactions between the two phases, entrained droplets will alter the flow field within the compressor, making the compressor operation deviate from the design conditions and thus decreasing compressor performance, so 1\% liquid entrainment may result in more than $1 \%$ reduction on system COP [15]. From Figure 11, it can be seen that at 600 RT, the relative difference between the tested and calculated COPs is very small $(<0.4 \%)$. This is because at $600 \mathrm{RT}$ the entrainment factor $E_{f g}$ increased by only about $0.3 \%$ at the end of charging (see Figure 8 ). At $700 \mathrm{RT}, E_{f g}$ increased by $0.87 \%$ at the end of charging, resulting in a $1.2 \%$ decrease in test COP relative to the calculated COP. As can be seen from Figure 11, a large deviation starts from the 5 th point because $E_{f g}$ at this point is relatively large $\left(E_{f g}=0.89 \%\right)$ and $E_{f g}$ increases rapidly with the increase of charge at 700 RT (see Figure 8), leading to the COP test value drops significantly at this point. At 800 RT, $E_{f g}$ increased by $1.9 \%$ at the end of charging, which made the test COP decreased by $2.5 \%$ relative to the calculated COP. It can be seen from Figure 11 that the test COP has a significant decrease from the calculated value at point $4\left(E_{f g}=1.02 \%\right)$. In view of the important influence of $E_{f g}$ on the unit COP, it is recommended that the maximum $E_{f g}$ for efficient operation of the unit be controlled at less than $1 \%$.

\section{Conclusions}

In this paper, an energy balance method for measuring the liquid entrainment of the evaporator of a water-cooled centrifugal chiller is proposed, including Method 1 (Equation (14)) involving only the heat exchange capacity of the evaporator and Method 2 (Equation (15)) involving the heat exchange capacity of both evaporator and condenser. This method is suitable for the measurement of the evaporator liquid entrainment of a single-stage centrifugal chiller with a certain degree of subcooling at the outlet of the condenser. Experimental measurement and uncertainty analysis were carried out on the liquid entrainment of a flooded evaporator under AHRI full-load conditions, with the experimental cooling capacities of 600,700 , and 800 RT and the refrigerant charge of $519-612 \mathrm{~kg}$. The results show that for a system with good thermal balance, the entrainment factor obtained by methods one and two are close to each other and Method 2 is recommended as it has higher accuracy. Uncertainty propagation analysis shows that for Method 2, the inlet and outlet water temperatures of the evaporator and condenser, motor input power, motor efficiency, transmission power loss and compressor suction and discharge temperatures are important factors to the uncertainty of the entrainment factor.

The experimental results show that the variation of the evaporator entrainment factor with refrigerant charge amount is different under different cooling capacity. At 600 RT, the increase of $E_{f g}$ is smooth with the increase of charge, while at 700 and 800 RT, the initial increase is slow and the subsequent increase is faster as charge increases. For the centrifugal chiller tested, the main factor causing the drop in COP is the liquid entrainment. At 700 and 800 RT, when the entrainment factor reaches $0.89 \%$ and $1.02 \%$, respectively, COP decreases significantly with the increase of charge. It is suggested that the maximum entrainment factor for efficient operation of the unit be controlled within $1 \%$.

Author Contributions: Conceptualization, X.H.; methodology, X.H.; software, X.H. and Z.L.; formal analysis, X.H.; investigation, X.H., Z.L. and Y.Z.; resources, X.H. and Y.Z.; data curation, X.H.; writing-original draft preparation, X.H.; writing-review and editing, All authors; visualization, X.H.; supervision, X.H. and Y.L. All authors have read and agreed to the published version of the manuscript.

Funding: This research received no external funding. 
Conflicts of Interest: The authors declare no conflict of interest.

\section{Nomenclature}

$A_{e} \quad$ heat transfer area of evaporator, $\left(\mathrm{m}^{2}\right)$

$c_{c w} \quad$ specific heat of cooling water, $\left(\mathrm{kJ} \cdot \mathrm{kg}^{-1} \cdot \mathrm{K}^{-1}\right)$

$c_{\text {ew }} \quad$ specific heat of cold water, $\left(\mathrm{kJ} \cdot \mathrm{kg}^{-1} \cdot \mathrm{K}^{-1}\right)$

$E_{f g} \quad$ entrainment factor

$f \quad$ polytropic work factor

$h_{0, \text { evo }} \quad$ total enthalpy of refrigerant vapor at evaporator outlet, $\left(\mathrm{kJ} \cdot \mathrm{kg}^{-1}\right)$

$h_{0, \text { elo }} \quad$ total enthalpy of refrigerant liquid at evaporator outlet, $\left(\mathrm{kJ} \cdot \mathrm{kg}^{-1}\right)$

$h_{\text {sub }} \quad$ enthalpy of refrigerant liquid at condenser outlet, $\left(\mathrm{kJ} \cdot \mathrm{kg}^{-1}\right)$

$h_{0, \text { cvo }} \quad$ total discharge enthalpy of compressor, $\left(\mathrm{kJ} \cdot \mathrm{kg}^{-1}\right)$

$h_{\text {cvo }} \quad$ discharge enthalpy of compressor, $\left(\mathrm{kJ} \cdot \mathrm{kg}^{-1}\right)$

$h_{\text {evo }} \quad$ vapor enthalpy at evaporator outlet, $\left(\mathrm{kJ} \cdot \mathrm{kg}^{-1}\right)$

$h_{\text {elo }} \quad$ saturated liquid enthalpy at evaporator outlet, $\left(\mathrm{kJ} \cdot \mathrm{kg}^{-1}\right)$

$K \quad$ total heat transfer coefficient of evaporator, $\left(\mathrm{W} \cdot \mathrm{m}^{-2} \cdot \mathrm{K}^{-1}\right)$

Load load factor of motor

$m_{l} \quad$ liquid flow rate from evaporator, $\left(\mathrm{kg} \cdot \mathrm{s}^{-1}\right)$

$m_{r} \quad$ refrigerant flow rate, $\left(\mathrm{kg} \cdot \mathrm{s}^{-1}\right)$

$m_{v} \quad$ vapor flow rate from evaporator, $\left(\mathrm{kg} \cdot \mathrm{s}^{-1}\right)$

$p_{c} \quad$ condensation pressure, $(\mathrm{kPa})$

$p_{\text {dis }} \quad$ discharge pressure, $(\mathrm{kPa})$

$p_{e} \quad$ evaporation pressure, $(\mathrm{kPa})$

$p_{\text {suct }} \quad$ suction pressure, $(\mathrm{kPa})$

$\Delta p_{c w} \quad$ cooling water pressure drop, $(\mathrm{kPa})$

$\Delta p_{\text {ew }} \quad$ cold water pressure drop, $(\mathrm{kPa})$

$Q_{c} \quad$ heat exchange capacity of condenser, $(\mathrm{kW})$

$Q_{e} \quad$ cooling capacity of evaporator, $(\mathrm{kW})$

$Q_{\text {mech }} \quad$ transmission power loss, $(\mathrm{kW})$

$Q_{\text {motor }}$ heat dissipation rate of motor, $(\mathrm{kW})$

$Q_{\text {oil }} \quad$ heat dissipation rate of oil cooler, $(\mathrm{kW})$

TB thermal balance of chiller

$t_{c w i} \quad$ cooling water inlet temperature, $\left({ }^{\circ} \mathrm{C}\right)$

$t_{c w o} \quad$ cooling water outlet temperature, $\left({ }^{\circ} \mathrm{C}\right)$

$t_{\text {dis }} \quad$ discharge temperature, $\left({ }^{\circ} \mathrm{C}\right)$

$t_{e} \quad$ evaporation temperature, $\left({ }^{\circ} \mathrm{C}\right)$

$t_{\text {ewi }} \quad$ cold water inlet temperature, $\left({ }^{\circ} \mathrm{C}\right)$

$t_{\text {ewo }} \quad$ cold water outlet temperature, $\left({ }^{\circ} \mathrm{C}\right)$

$t_{l o} \quad$ condenser outlet liquid temperature, $\left({ }^{\circ} \mathrm{C}\right)$

$t_{\text {suct }} \quad$ suction temperature, $\left({ }^{\circ} \mathrm{C}\right)$

$\Delta t \quad$ logarithmic mean temperature difference of evaporator, $\left({ }^{\circ} \mathrm{C}\right)$

$u_{c v o} \quad$ vapor velocity at compressor outlet, $\left(\mathrm{m} \cdot \mathrm{s}^{-1}\right)$

$u_{\text {elo }} \quad$ droplet velocity at evaporator outlet, $\left(\mathrm{m} \cdot \mathrm{s}^{-1}\right)$

$u_{\text {evo }} \quad$ vapor velocity at evaporator outlet, $\left(\mathrm{m} \cdot \mathrm{s}^{-1}\right)$

$v_{\mathcal{C}} \quad$ specific volume of refrigerant vapor in the condenser, $\left(\mathrm{m}^{3} \cdot \mathrm{kg}^{-1}\right)$

$V_{c w} \quad$ volume flow rate of cooling water, $\left(\mathrm{m}^{3} \cdot \mathrm{s}^{-1}\right)$

$v_{e} \quad$ specific volume of refrigerant vapor in the evaporator, $\left(\mathrm{m}^{3} \cdot \mathrm{kg}^{-1}\right)$

$V_{e w} \quad$ volume flow rate of cold water, $\left(\mathrm{m}^{3} \cdot \mathrm{s}^{-1}\right)$

$V_{n} \quad$ rated volumetric flow rate of compressor, $\left(\mathrm{m}^{3} \cdot \mathrm{s}^{-1}\right)$

$V_{s} \quad$ volumetric flow rate at evaporator state, $\left(\mathrm{m}^{3} \cdot \mathrm{s}^{-1}\right)$

$W_{\text {comp }}$ compressor input power, $(\mathrm{kW})$

$W_{\text {in }} \quad$ motor input power, $(\mathrm{kW})$

$w_{n} \quad$ rated specific polytropic work of compressor, $\left(\mathrm{kJ} \cdot \mathrm{kg}^{-1}\right)$

$\eta \quad$ compressor efficiency factor

$\eta_{c} \quad$ compressor efficiency

$\eta_{e} \quad$ motor efficiency 


$\begin{array}{ll}\eta_{\text {mech }} & \text { transmission efficiency } \\ \eta_{n} & \text { rated compressor efficiency } \\ \rho_{c w} & \text { cooling water density, }\left(\mathrm{kg} \cdot \mathrm{m}^{-3}\right) \\ \rho_{e w} & \text { cold water density, }\left(\mathrm{kg} \cdot \mathrm{m}^{-3}\right) \\ \Phi & \text { volumetric flow percent } \\ \Psi & \text { polytropic work percent }\end{array}$

\section{References}

1. Al-Sarkhi, A.; Sarica, C.; Qureshi, B. Modeling of droplet entrainment in co-current annular two-phase flow: A new approach. Int. J. Multiph. Flow 2012, 39, 21-28. [CrossRef]

2. Pan, L.; Hanratty, T.J. Correlation of entrainment for annular flow in horizontal pipes. Int. J. Multiph. Flow 2002, $28,385-408$. [CrossRef]

3. Pan, L.; Hanratty, T.J. Correlation of entrainment for annular flow in vertical pipes. Int. J. Multiph. Flow 2002, $28,363-384$. [CrossRef]

4. Sawant, P.; Ishii, M.; Mori, M. Droplet entrainment correlation in vertical upward co-current annular two-phase flow. Nucl. Eng. Des. 2008, 238, 1342-1352. [CrossRef]

5. Welter, K.; Wu, Q.; You, Y.; Abel, K.; McCreary, D.; Bajorek, S.; Reyes, J. Experimental investigation and theoretical modeling of liquid entrainment in a horizontal tee with a vertical-up branch. Int. J. Multiph. Flow 2004, 30, 1451-1484. [CrossRef]

6. Meng, Z.; Fu, X.; Ding, L.; Dong, B.; Tian, W.; Yang, Y.; Su, G. Experimental and theoretical investigation of liquid entrainment through small-scaled ADS-4 in AP1000. Exp. Therm. Fluid Sci. 2014, 57, 177-187. [CrossRef]

7. Sun, D.; Zhang, Y.; Qiu, S.; Zan, Y.; Liu, W.; Su, G. Models development of liquid drops entrainment at a T-junction with a large vertical up branch. Int. J. Heat Mass Transf. 2017, 110, 555-561. [CrossRef]

8. Kataoka, I.; Ishii, M. Mechanistic modeling of pool entrainment phenomenon. Int. J. Heat Mass Transf. 1984, 27, $1999-2014$.

9. Sun, D.; Xiang, Y.; Tian, W.; Liu, J.; Zhang, P.; Qiu, S.; Su, G. Experimental investigation of upper plenum entrainment in AP1000. Prog. Nucl. Energy 2014, 80, 80-85. [CrossRef]

10. Zhang, P.; Li, W.; Di, Z.; Hu, X.; Chen, L.; Chang, H.; Chen, P. An experimental study of pool entrainment with side exit. Ann. Nucl. Energy 2017, 110, 406-411. [CrossRef]

11. Lu, M.; Xie, H. Experimental and theoretical investigation on pool entrainment under small air flow rate. Exp. Therm. Fluid Sci. 2019, 105, 35-46. [CrossRef]

12. Asher, W.; Eckels, S.J. Characterization of liquid refrigerant R-123 flow emerging from a flooded evaporator tube bundle. Sci. Technol. Built Environ. 2018, 24, 1026-1038. [CrossRef]

13. Asher, W.; Eckels, S.J. Characterization and numerical simulation of liquid refrigerant R-134a flow emerging from a flooded evaporator tube bundle. Int. J. Refrig. 2019, 107, 275-287. [CrossRef]

14. Anish, S.; Kim, H.D. Effects of Wet Compression on the Flow Behavior of a Centrifugal Compressor: A CFD Analysis. In Proceedings of the ASME Turbo Expo 2014: Turbine Technical Conference and Exposition, Dusseldorf, Germany, 16-20 June 2014; pp. V02DT42A002-V02DT42A.

15. Halbe, C.V.; O’Brien, W.F.; Cousins, W.T.; Sishtla, V. (Eds.) A Numerical Analysis of the Effects of Liquid Carryover on the Performance of a Two-Stage Centrifugal Compressor. In Proceedings of the ASME Turbo Expo 2018: Turbomachinery Technical Conference and Exposition, Oslo, Norway, 11-15 June 2018; p. V02BT44A028.

16. AHRI. Standard 550/590, Performance Rating of Water-Chilling and Heat Pump Water-Heating Packages Using the Vapor Compression Cycle; Air-Conditioning and Refrigeration Institute: Arlington, VA, USA, 2018; p. 8.

17. Moran, M.J.; Shapiro, H.N.; Boettner, D.D.; Bailey, M.B. Fundamentals of Engineering Thermodynamics, 7th ed.; John Wiley \& Sons, Inc.: Hoboken, NJ, USA, 2010; p. 555.

18. Zhang, C.L.; Yang, L.; Shao, L.L. Refrigeration and Air-Conditioning System Modeling and Analysis Using Greatlab; Chemical Industry Press: Beijing, China, 2015; p. 57.

19. Moffat, R.J. Describing the uncertainties in experimental results. Exp. Therm. Fluid Sci. 1988, 1, 3-17. [CrossRef]

20. Klein, S.A. Engineering Equation Solver; Version 10.809-3D; (1992-2020; F-Chart Software, LLC: Madison, WI, USA, 2000. Available online: https: / / fchartsoftware.com/ees/ (accessed on 2 September 2021).

21. Lemmon, E.W.; Huber, M.L.; McLinden, M.O. NIST Standard Reference Database 23: Reference Fluid Thermodynamic and Transport Properties-Refprop, Version 9.1; National Institute of Standards and Technology, Standard Reference Data Program: Gaithersburg, MD, USA, 2013.

22. ASHRAE. 2012 ASHRAE Handbook HVAC Systems and Equipment (SI Edition); ASHRAE: Atlanta, GA, USA, 2012; pp. 38.27-38.32. 